\title{
Oxigenação extracorpórea por membrana em cães sem auxílio circulatório
}

\author{
Pumpless extracorporeal membrane oxygenation in dogs
}

\author{
Alessandra Castello da Costa ${ }^{\mathrm{I}}$ Lara Lages da Silveira ${ }^{\mathrm{II}}$ Felipp da Silveira Ferreira ${ }^{\mathrm{II}}$ \\ Cíntia Lourenço Santos ${ }^{\mathrm{II}}$ Edmundo Jorge Abílio ${ }^{\mathrm{I}}$ Fernanda Antunes ${ }^{\mathrm{III}}$ \\ Antônio Peixoto Albernaz ${ }^{\text {II }}$ Cláudio Baptista de Carvalho ${ }^{\text {III }}$ \\ Giseli dos Santos Ferreira ${ }^{\mathrm{II}}$ Daniela Fantini Vale ${ }^{\mathrm{III}}$ Josias Alves Machado ${ }^{\mathrm{III}}$ \\ Guilherme Alexandre Soares Monteiro ${ }^{\mathrm{III}}$ André Lacerda de Abreu Oliveira ${ }^{\mathrm{II}}{ }^{*}$
}

RESUMO

A oxigenação extracorpórea por membrana com desvio arterio-venoso (AV-ECMO), em seres humanos, é uma técnica de suporte à vida capaz de assumir em parte ou totalmente as funções dos pulmões, quando estes já não são capazes de promover uma adequada hematose. Para conhecer a viabilidade deste método em cães (Canis familiaris), o presente estudo aplicou a AV-ECMO em dez indivíduos adultos, sem raça definida, com uma expectativa de três horas de duração para cada experimento. No grupo $1(n=4)$, ventilação mecânica e infusão contínua de dopamina foram associadas ao suporte extracorpóreo. No grupo $2(n=6)$, nenhuma medida de terapia intensiva foi instituída concomitantemente à AV-ECMO. O comportamento dos parâmetros de gasometria e os níveis de lactato foram avaliados a cada 10 minutos e 1 hora, respectivamente, por meio de da avaliação de amostras sanguíneas arteriais. O suporte inotrópico foi capaz de promover uma melhor perfusão tecidual com sangue oxigenado, sugerida pelos níveis de lactato e pressão arterial média mensurados, assim como a hemogasometria revelou melhores resultados quando a ventilação mecânica foi utilizada simultaneamente à AV-ECMO. Portanto, aplicada conjuntamente com suporte da terapia intensiva, a AV-ECMO é uma técnica viável para o uso em cães com falência respiratória, refratária aos tratamentos convencionais.

Palavras-chave: oxigenadores de membrana, gasometria, falência respiratória, cães.

\section{ABSTRACT}

In humans, arteriovenous extracorporeal membrane oxygenation (AV-ECMO) is a life support technique capable to assume in part or totally lungs functions, when these are not able to promote appropriated hematosis. To know the viability of this method in dogs (Canis familiaris), the present study applied AVECMO in ten adult mixed-breed dogs, with expected three hours of duration to each experiment. In Group $1(n=4)$, mechanical ventilation and dopamine continuous infusion were associated with the extracorporeal support. In Group $2(n=6)$, any other measure of intensive care was used concomitantly AV-ECMO. Blood gas analysis and lactate levels were evaluated every 10 minutes and one hour, respectively, from arterial blood samples. Inotropic support improved the blood flow through the circuit promoting a better tissue perfusion with oxygenated blood, suggested by the lactate and mean arterial pressure levels measured. Mechanical ventilation used simultaneously with AV-ECMO was able to further improve levels of blood gases. Therefore, if applied along intensive care support, AV-ECMO is a viable technique for use in dogs with respiratory failure refractory to conventional treatments.

Key words: membrane oxygenators, blood gas analysis, respiratory failure, dogs.

\section{INTRODUÇÃO}

A falência respiratória (FR) é caracterizada por uma profunda deterioração na oxigenação e/ou ventilação, apesar do emprego de terapia de suporte respiratória. A mortalidade para pacientes humanos nestas condições encontra-se acima de 66\% (NEHRA et al., 2009). Quando a FR é associada com um risco de mortalidade acima de $80 \%$ (COOK, 2004) e quando os tratamentos convencionais não obtêm sucesso (ANKE et al., 2009), a Medicina ainda conta com a possibilidade de se realizar a troca gasosa sanguínea fora dos pulmões (PETROU et al., 2006), permitindo que haja tempo para o tratamento das afecções de base e a resolução do processo pulmonar

'Universidade Estadual do Norte Fluminense Darcy Ribeiro (UENF), Campos dos Goytacazes, RJ, Brasil.

"Universidade Federal do Rio de Janeiro (UFRJ), Rio de Janeiro, RJ, Brasil.

IIIUnidade de Experimentação Animal (UEA), UENF, Av. Alberto Lamego, 2.000, 28013-602, Campos dos Goytacazes, RJ, Brasil. E-mail: andrevet@uenf.br.*Autor para correspondência. 
(BARTLETT et al., 1986). Essa técnica de suporte promove um desvio cardiopulmonar e é conhecida como oxigenação extracorpórea por membrana (ECMO), cujos resultados em seres humanos alcançam um índice de sobrevivência de $77 \%$ em casos de extrema gravidade (ANKE et al., 2009).

Recentemente, houve um grande crescimento na demanda por esta tecnologia, devido às complicações pulmonares promovidas pela infecção com o vírus da influenza A (H1N1). Alguns indivíduos desenvolvem a síndrome da angústia respiratória aguda (SARA) e requerem uma terapia de suporte mais efetiva. Tratados com a ECMO, estes pacientes, em geral adultos jovens, com hipoxemia grave, apresentam um índice de mortalidade de $21 \%$, o que é considerado um bom resultado para quadros de tamanha gravidade (DAVIES et al., 2009). Diversos casos de contaminação pelo vírus H1N1 já foram confirmados em animais de companhia, como cães, gatos e ferrets, e alguns deles, como os seres humanos, também desenvolvem complicações pulmonares promovidas por este agente etiológico (2011 H1N1, 2012).

Problemas respiratórios graves de outra natureza são comuns em cães. Apesar da oxigenação extracorpórea por membrana ser considerada um método avançado de suporte à vida, é de grande complexidade técnica e sua utilização ainda é escassa na Medicina Veterinária. Resultados experimentais com cães indicam que a ECMO possa beneficiar esses animais com afecções pulmonares, como trauma, edema e outras doenças que comprometam a hematose, associadas à refratariedade dos tratamentos convencionais (COSTA et al., 2008).

Entre os três desvios cardiopulmonares possíveis para a ECMO, veno-venoso, venoarterial e artério-venoso, este último envolve menos complicações operacionais do que os demais (TOTAPALLY et al., 2004), já que, fora o suprimento de oxigênio, o sistema não requer fontes adicionais de energia, como a máquina de circulação extracorpórea (ZIMMERMANN et al., 2007). É o gradiente de pressão sanguínea que impulsiona o sangue através do circuito (KOPP et al., 2006), sendo o coração a bomba pressora. Por isso, a estabilidade cardiovascular é essencial para promover um fluxo sanguíneo suficiente através da unidade de troca gasosa (ZIMMERMANN et al., 2007).

O objetivo deste estudo foi avaliar a viabilidade do uso da AV-ECMO em cães com e sem auxílio de fármaco inotrópico e ventilação mecânica, analisando-se, especificamente, o comportamento de parâmetros da hemogasometria, dos níveis de lactato e da pressão arterial média (PAM) durante o procedimento. A hipótese proposta é que a AV-ECMO seja um método de suporte à vida tecnicamente viável para ser implementada na rotina das unidades de terapia intensiva em casos de cães com FR, quando há refratariedade das terapias convencionais.

\section{MATERIAL E MÉTODOS}

Foram estudados dez cães adultos, sem raça definida, machos e fêmeas indistintamente, saudáveis, pesando $16,98 \pm 8,84 \mathrm{~kg}$. Imediatamente após o término do experimento, todos os indivíduos foram submetidos à eutanásia por meio da administração intravenosa de pentobarbital sódico, na dose de $120 \mathrm{mg}$ para os primeiros $4,5 \mathrm{~kg}$ e $60 \mathrm{mg}$ para os demais, respeitando-se os princípios éticos do uso de animais para experimentação.

Uma amostra de sangue total arterial foi coletada da artéria femoral direita de todos os animais, já anestesiados, para a obtenção de valores de referência de controle dos níveis de lactato e dos parâmetros de hemogasometria basais do paciente, assim como também foi aferida a pressão arterial média de forma invasiva, na artéria femoral, por meio de um monitor multiparamétrico.

Uma amostra de sangue venoso foi coletada para realização do hematimetria, hemoglobinometria, hematócrito, leucograma, testes de coagulação, provas de função hepática e renal e a contagem plaquetária.

A medicação pré-anestésica consistiu na associação de acepromazina $\left(0,05 \mathrm{mg} \mathrm{kg}^{-1}\right)$ e sulfato de morfina $\left(0,1 \mathrm{mg} \mathrm{kg}^{-1}\right)$, pela via subcutânea. A indução anestésica foi alcançada por meio da administração intravenosa de cloridrato de cetamina $\left(5 \mathrm{mg} \mathrm{kg}^{-1}\right)$ e diazepan $\left(0,5 \mathrm{mg} \mathrm{kg}^{-1}\right)$. Para a manutenção anestésica, utilizou-se a

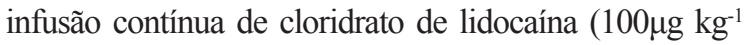
$\left.\min ^{-1}\right)$, fentanil $\left(10 \mu \mathrm{g} \mathrm{kg}^{-1} \mathrm{~h}^{-1}\right)$ e cloridrato de cetamina ( $5 \mu \mathrm{g}$ $\mathrm{kg}^{-1} \min ^{-1}$ ) em bomba de infusão volumétrica.

A incoagulabilidade sanguínea foi estabelecida pela administração de heparina sódica na solução inicial contida no oxigenador de membranas, composto de 2000UI diluídas em $110 \mathrm{~mL}$ de solução de Ringer com lactato (conforme proposto por BALAGANGADHAR et al., 2004) e por via intravenosa, de $350 \mathrm{UI} \mathrm{k^{-1 }}$ a cada hora, logo após a canulação da artéria e da veia femorais. Imediatamente antes de se iniciar o desvio cardiopulmonar, todos os animais receberam, intravenosamente, cloreto de succinilcolina $\left(0,1 \mathrm{mg} \mathrm{kg}^{-1}\right)$ para promover a apneia.

Após a canulação da artéria e da veia femorais com cateteres siliconizados de aproximadamente $3 / 4$ do diâmetro do vaso, e estes 
conectados ao oxigenador de membranas capilar infantil, iniciou-se um desvio cardiopulmonar passivo. O sangue, portanto, ao atravessar o pulmão artificial, capaz de oxigená-lo e remover o $\mathrm{CO}_{2}$, retornava ao corpo através da veia femoral. Este circuito conta somente com o coração do paciente como a bomba pressora do sistema.

O oxigenador de membranas descartável recebendo $\mathrm{FiO}_{2}$ de $100 \%$ e fluxo de $\mathrm{O}_{2}$ de $4 \mathrm{~L} \mathrm{~min}^{-1}$ - foi disposto na mesa em que se encontrava o paciente, ambos permanecendo na mesma altura para não aumentar a resistência ao fluxo sanguíneo através do circuito (Figura 1).

No grupo $1(n=4)$, foram utilizadas medidas de terapia intensiva concomitantemente à AV-ECMO: após a intubação traqueal, houve suporte ventilatório mínimo, com 12 movimentos respiratórios por minuto, PEEP de $3 \mathrm{cmH}_{2} \mathrm{O}$ e $\mathrm{FiO}_{2}$ de $21 \%$ a um fluxo inspirado de oxigênio de $3 \mathrm{Lmin}^{-1}$. Assim, para minimizar as chances de surgimento de instabilidade hemodinâmica e auxiliar o fluxo sanguíneo e a perfusão tecidual, foi realizada infusão contínua de dopamina, na dose de $5 \mu \mathrm{g} \mathrm{kg}^{-1} \mathrm{~min}^{-1}$.

Os animais do grupo $2(n=6)$ não receberam suporte inotrópico ou ventilação mecânica. $\mathrm{O}$ intuito foi simular o efeito exclusivo da AV-ECMO no organismo, sendo esta a única responsável pela oxigenação sanguínea destes animais.

A duração prevista da AV-ECMO para todos os cães foi de três horas. Uma amostra sanguínea total arterial foi coletada imediatamente após a indução da apneia e a cada 10 minutos, até o final do experimento, para a verificação de parâmetros de hemogasometria do animal. O lactato foi avaliado uma, duas e três horas após o início da oxigenação extracorpórea, e a pressão arterial média aferida em intervalos de 10 minutos.

A análise estatística foi realizada com o auxílio de um software comercial (SPSS for Windows 11.0.1). Os resultados foram expressos em média \pm desvio-padrão, sendo considerados significativos se $\mathrm{P}<0,05$.

\section{RESULTADOS E DISCUSSÃO}

Os resultados médios obtidos para o eritrograma mantiveram-se estáveis durante as três horas de ECMO. Especificamente para a variável hematócrito no G1, o valor pré-ECMO foi de 29, $66 \%$, mantendo-se em 30,82\% nos que atingiram uma hora e meia de experimento (T4) e ficando próximo deste valor nos que atingiram três horas (T4), 31,5\%. No $\mathrm{G} 2$, o hematócrito médio pré-ECMO era de $28,55 \%$ e manteve-se estável durante todo o experimento $(26,68$ e $29,9 \%$ ) com uma hora e meia e três horas de ECMO, respectivamente. Em uma análise inicial, acredita-se que, por ser imediatamente após o início da ECMO (T1 = cinco minutos), possa ter ocorrido uma hemodiluíção transitória quando o sangue entrou em contato com a solução presente no priming. Não houve alterações significativas no valores da hemoglobina.

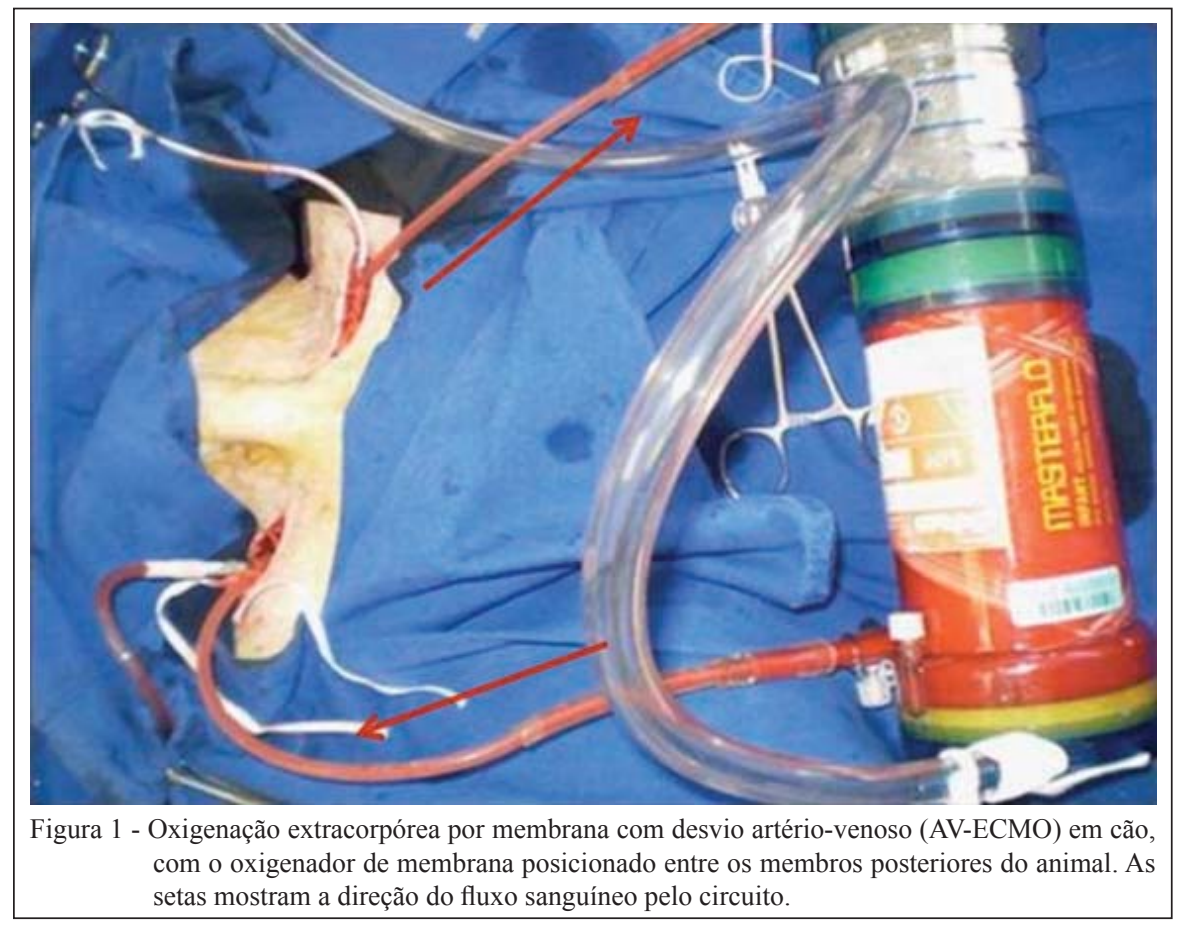

Ciência Rural, v.44, n.5, mai, 2014. 
Com base nos valores médios obtidos para plaquetometria em G1 (entre $363.000 \mu \mathrm{l}^{-1}$ no tempo 0 e $305.666 \mu \mathrm{l}^{-1}$ no tempo 7) e G2 (entre $354.500 \mu \mathrm{l}^{-1}$ no tempo 0 e $220.500 \mu \mathrm{l}^{-1}$ no tempo 7), percebe-se que não houve diferença estatística significativa durante as três horas de ECMO.

Analisando os dados entre ambos os grupos para os marcadores ALT, AST, FA, Ureia e Creatinina em todos os tempos propostos, verifica-se que não houve diferença significativa entre os resultados obtidos.

Os testes de coagulação ativados, realizados a cada 15 minutos durante a ECMO, mostraram-se dentro dos parâmetros desejáveis de anticoagulação.

Os animais de ambos os grupos apresentavam condições hemogasométricas arteriais estatisticamente semelhantes antes do início dos experimentos, exceto em relação ao $\mathrm{pCO}_{2}$, segundo a ANOVA dos valores de controle (Tabela 1). Os cães do primeiro grupo, embora diante de valores ainda normais de $\mathrm{pH}$, apresentavam $\mathrm{HCO}_{3-}$ e EB alterados, demonstrando uma tendência ao desenvolvimento de acidose metabólica, enquanto, nos cães do segundo grupo, este distúrbio já se encontrava instalado. FERREIRA et al. (2005), lembram que o aparecimento de acidose metabólica no período préoperatório pode estar relacionado ao jejum. Todos os animais foram submetidos ao jejum hídrico de 4 horas, e jejum alimentar de, pelo menos, 15 horas no momento do início dos procedimentos.

Durante a AV-ECMO, o grupo 1 apresentou um leve declínio do $\mathrm{pH}$ e da $\mathrm{pCO}_{2}$, além da diminuição dos valores de $\mathrm{HCO}_{3-}$ e EB. A redução da $\mathrm{pCO}_{2}$ pode estar associada à utilização concomitante da ventilação mecânica e do fluxo de $\mathrm{O}_{2}$ para o oxigenador de membrana. Este último, segundo ZIMMERMANN et al. (2007), é capaz de remover toda a produção metabólica de $\mathrm{CO}_{2}$, além de oxigenar o sangue. No entanto, não foi o que se observou neste estudo, já que os valores da $\mathrm{pCO}_{2}$ diferiram estatisticamente em ambos os grupos. Os animais que não receberam ventilação mecânica (grupo 2) apresentaram níveis mais elevados e fora da faixa de normalidade, o que sugere que o fluxo de oxigênio que chegava ao oxigenador de membranas $\left(4 \mathrm{~L} \mathrm{~min}^{-1}\right)$ não era capaz de remover o $\mathrm{CO}_{2}$ de maneira adequada. PERALTA et al. (2005), por sua vez, descreveram a utilização de um fluxo de oxigênio de 10 a $12 \mathrm{~L} \mathrm{~min}^{-1}$., obtendo os efeitos esperados.

Tabela 1 - Comportamento das variáveis de hemogasometria em relação ao tempo (em horas) apresentados pelos cães dos grupo 1 e 2 antes e durante a AV-ECMO.

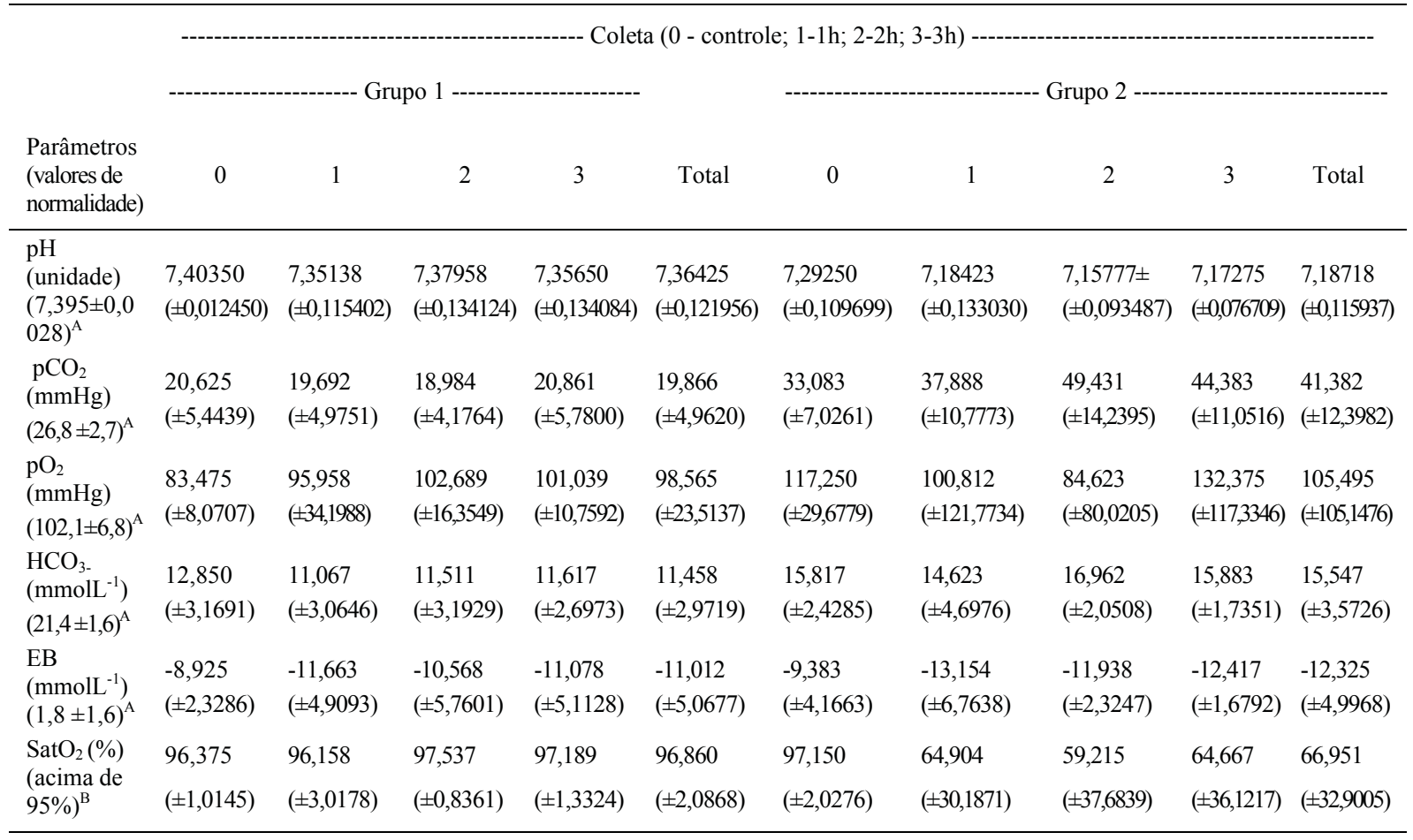

A - Segundo ILKIW et al. (1991). B - Segundo SMITH \& TAYLOR (2008). C - Segundo DAY (2002).

Ciência Rural, v.44, n.5, mai, 2014. 
O grupo 2 apresentou uma queda acentuada no $\mathrm{pH}$ e na $\mathrm{SatO}_{2}$, reduzindo-se também os valores de $\mathrm{HCO}_{3-}$ e $\mathrm{EB}$ e agravando ainda mais a acidose metabólica pré-existente. Devido à elevação dos níveis de $\mathrm{pCO}_{2}$, observou-se uma acidose respiratória associada, contribuindo para a promoção de um distúrbio eletrolítico misto. No entanto, é particularmente interessante observar que, apesar dos piores resultados da $\mathrm{SatO}_{2}$ apresentados pelo grupo 2, este parâmetro manteve-se em níveis ótimos durante todo o tempo no animal $6(97,38 \pm 2,76 \%)$, demonstrando que a AVECMO sozinha é capaz de promover uma adequada saturação de oxigênio no sangue, embora outros fatores estejam essencialmente relacionados ao sucesso da terapia, como, por exemplo, a utilização concomitante de agente inotrópico para manter a estabilidade hemodinâmica do paciente e os níveis de hemoglobina no sangue. Durante a AV-ECMO, os parâmetros $\mathrm{pH}, \mathrm{pCO}_{2}, \mathrm{HCO}_{3-}$ e $\mathrm{SatO}_{2}$ apresentados por ambos os grupos foram considerados, pela ANOVA, estatisticamente diferentes.

Outro ponto a ser abordado é o fato de que, segundo ZIMMERMANN et al. (2006) e KOOP et al. (2006), a transferência de oxigênio no desvio artériovenoso pode não ser suficiente justamente porque o sangue que chega ao oxigenador é o arterial, sendo este desvio cardiopulmonar capaz de promover apenas um aumento moderado da oxigenação. Porém, como os animais do grupo 2 não receberam ventilação mecânica e apresentavam-se em apneia, o sangue que alcançava o oxigenador possuía características venosas e, teoricamente, deveria ser capaz de alcançar um nível de saturação alto, como se pôde comprovar com os dados apresentados pelo animal 6, cuja $\mathrm{SatO}_{2}$ foi excelente.

Dos quatro animais do grupo $1,75 \%$ sobreviveram aos 180 minutos previstos de experimento (Tabela 2), corroborando os resultados obtidos nos serviços de ECMO na medicina, que, segundo PERALTA et al. (2005), encontram-se entre $50 \%$ e $80 \%$ nos centros de maior experiência. Os animais do primeiro grupo receberam auxílio de medidas de terapia intensiva, como a ventilação mecânica e a administração de fármaco inotrópico, à semelhança dos protocolos adotados para a instituição da AV-ECMO em seres humanos (ZIMMERMANN et al., 2006). Este parece ser o ponto-chave para a avaliação dos resultados apresentados por ambos os grupos ao longo do estudo.

Segundo ZICK et al. (2006), e MUELLENBACH et al. (2008), uma PAM de 70mmHg é mínimo desejável para assegurar um fluxo sanguíneo adequado por todo o circuito da AV-ECMO, pois, entre os quatro animais do primeiro grupo, três permaneceram em AV-ECMO durante todos os 180 minutos previstos, com PAM em níveis ótimos, excetuando-se o animal 1. Neste animal, cuja PAM alcançou níveis extremamente diminuídos ( $48 \pm 14 \mathrm{mmHg}$ ) e, portanto, inadequados para a manutenção de um desvio extracorpóreo arteriovenoso, sem o auxílio de máquina de circulação extracorpórea. Ele apresentava, antes de instituído o desvio cardiopulmonar, PAM de $68 \mathrm{mmHg}$, inferior ao mínimo desejado.

Os animais do segundo grupo obtiveram os piores índices de sobrevivência no estudo (33\%). Como era esperado, pelo não uso de agente inotrópico, os valores de PAM obtidos foram inferiores aos do grupo 1, apesar dos oxigenadores terem sido posicionados na mesma altura dos animais para facilitar a ação da gravidade, conforme descreveu BREDERLAU et al. (2004). Dos seis cães do grupo 2, dois apresentaram valores diminuídos de PAM durante o experimento. $\mathrm{O}$ animal 7 apresentou uma média de $76 \mathrm{mmHg}$, porém, com elevado desviopadrão. $\mathrm{O}$ de número 5 foi o único deste grupo que, apesar dos níveis de PAM excelentes, veio a óbito supostamente por coagulação intravascular disseminada. Apesar dos testes para sua confirmação não terem sido executados por estarem programados a serem realizados com 15 minutos o animal veio a óbito

Tabela 2 - Valores da pressão arterial média (PAM) apresentados pelos cães durante a oxigenação extracorpórea por membrana com desvio artério-venoso (AV-ECMO).

\begin{tabular}{|c|c|c|c|c|c|}
\hline Grupo & Animal & PAM (mmHg) controle & PAM (mmHg) durante & Óbito durante & Tempo (min) \\
\hline 1 & 1 & 68 & $48 \pm 14$ & Sim & 80 \\
\hline 1 & 2 & 95 & $113 \pm 26$ & Não & 180 \\
\hline 1 & 3 & 80 & $86 \pm 13$ & Não & 180 \\
\hline 1 & 4 & 67 & $87 \pm 19$ & Não & 180 \\
\hline 2 & 5 & 71 & $117 \pm 18$ & Sim & 10 \\
\hline 2 & 6 & 78 & $92 \pm 16$ & Não & 180 \\
\hline 2 & 7 & 90 & $76 \pm 25$ & Sim & 50 \\
\hline 2 & 8 & 70 & $61 \pm 17$ & Sim & 70 \\
\hline 2 & 9 & 79 & $69 \pm 12$ & Sim & 40 \\
\hline 2 & 10 & 87 & $95 \pm 22$ & Não & 180 \\
\hline
\end{tabular}

Ciência Rural, v.44, n.5, mai, 2014. 
com apenas 10 minutos de oxigenação extracorpórea, com a formação de coágulos sanguíneos nas cânulas e no oxigenador de membranas. O protocolo de anticoagulação adotado não foi eficaz neste animal, apesar de GOMES et al. (1993) terem administrado doses inferiores em caninos submetidos ao mesmo procedimento e pelo mesmo período, obtendo bons resultados. Esta é uma das principais complicações da ECMO, com risco de morte (KOPP et al., 2006).

Segundo ALLEN \& HOLM (2008), as mensurações de lactato podem monitorar o êxito de determinadas terapias, sendo este parâmetro considerado um bom marcador na detecção de hipoperfusão, já que o aumento de sua concentração correlaciona-se com a oxigenação inadequada dos tecidos. A comparação dos níveis de lactato do grupo 1 e do grupo 2 com seus respectivos valores de controle sugere que houve uma melhor perfusão tecidual com sangue oxigenado no primeiro grupo. No entanto, a ANOVA não revelou diferença estatística entre os animais de ambos os grupos. Apesar disso, os níveis de lactato atingidos pelo segundo grupo foram mais elevados, demonstrando uma pior perfusão tecidual com sangue oxigenado, o que vai ao encontro os piores valores de PAM obtidos nestes animais. Percebeu-se, também, que níveis ótimos de $\mathrm{Sat}_{\mathrm{O} 2}$ foram alcançados até um máximo de $4,3 \mathrm{mmol} \mathrm{L}^{-1}$ de lactato, apesar de os níveis desejados, segundo a literatura (DAY, 2002), serem inferiores a 2-2,5mmol $\mathrm{L}^{-1}$. A partir de $7,3 \mathrm{mmol} \mathrm{L}^{-1}$ é que a $\mathrm{Sat}_{\mathrm{O} 2}$ declinou demasiadamente, em níveis incompatíveis com a vida. Segundo CHEUNG \& FINER (1994), o lactato pode servir como um bom valor de prognóstico, sendo essa afirmação comprovada pela observação do comportamento desta variável no presente estudo.

\section{CONCLUSÃO}

A técnica de oxigenação extracorpórea estudada foi capaz de promover uma adequada saturação de oxigênio no sangue com e sem a ajuda da ventilação mecânica, sendo que esta ferramenta melhora ainda mais os níveis dos gases sanguíneos. Resultados mais animadores foram obtidos com o uso concomitante de ventilação mecânica e apoio inotrópico, Acredita-se, portanto, que a AV-ECMO seja uma técnica viável para aplicação em cães.

\section{REFERÊNCIAS}

www.avma.org/KB/Resources/Reference/Pages/2009-H1N1-FluVirus-Outbreak.aspx>. Acesso em: 16 jul. 2012.

ALLEN, S.E.; HOLM, J.L. Lactate: physiology and clinical utility. Journal of Veterinary Emergency and Critical Care, v.18, p.123-132, 2008. Disponível em: <http://onlinelibrary.wiley. com/doi/10.1111/j.1476-4431.2008.00286.x/abstract>. Acesso em: 8 out. 2012. doi: 10.1111/j.1476-4431.2008.00286.x.

ANKE, P.C. et al. Changes in buccal microcirculation following extracorporeal membrane oxygenation in term neonates with severe respiratory failure. Critical Care Medicine, v.37, p.11211124, 2009. Disponível em: <http://journals.lww.com/ccmjournal/ Abstract $/ 2009 / 03000 /$ Changes in buccal microcirculation following.45.aspx $>$. Acesso em: 8 out. 2012 . doi: 10.1097/ CCM.0b013e3181962a5f.

BALAGANGADHAR, R.T. et al. Cardiovascular stability during arteriovenous extracorporeal therapy: a randomized controlled study in lambs with acute lung injury. Critical Care, v.8, p.R495-R503, 2004. Disponível em: <http://ccforum.com/ content/8/6/R495>. Acesso em 21 maio, 2013. doi:10.1186/ cc2983.

BARTLETT, R.H. et al. Extracorporeal membrane oxygenation (ECMO) in neonatal respiratory failure: 100 cases. Annals of Surgery, v.204, p.236-244, 1986. Disponível em: <http://www.ncbi. nlm.nih.gov/pmc/articles/PMC1251270/>. Acesso em 8 out. 2012.

BREDERLAU, J. et al. Pumpless extracorporeal lung assist in severe blunt chest trauma. Journal of Cardiothoracic and Vascular Anesthesia, v.18, p.777-779, 2004. Disponível em: $<$ http://www.intertema.com.tr/tr/yapay/pdf/compendium/CE1_ PDFs_iLA/4.pdf>. Acesso em: 27 dez. 2009. doi: 10.1053/j. jvca. 2004.08 .022 .

CHEUNG, P.; FINER, N.N. Plasma lactate concentration as a predictor of death in neonates with severe hypoxemia requiring extracorporeal membrane oxygenation. Journal of Pediatrics, v.125, p.763-768, 1994. Disponível em: <http://www.sciencedirect. com/science/article/pii/S0022347606801812>. Acesso em: 27 dez. 2009. doi: 10.1016/S0022-3476(06)80181-2.

COOK, L.N. Update on extracorporeal membrane oxygenation. Paediatric Respiratory Reviews, v.5, p.S329-S337, 2004. Disponível em: <http://www.sciencedirect.com/science/article/pii/ S1526054204900589>. Acesso em: 8 out. 2012. doi: 10.1016/ S1526-0542(04)90058-9.

COSTA, A. et al. Oxigenação extracorpórea por membrana (ECMO) sem auxílio circulatório em canino. Medvep-Revista Científica de Medicina Veterinária, v.6, n.19, p. 293-300, 2008. Disponível em: $<$ http://www.medvep.com.br/index.php?pag=artigos\&pagina=18>. Acesso em: 8 out. 2012.

DAVIES, A. et al. Extracorporeal membrane oxygenation for 2009 influenza A (H1N1) acute respiratory distress syndrome. Journal American Medical Association, v.302, p.1888-1895, 2009. Disponível em: <http://jama.jamanetwork.com/article. aspx? articleid $=184800>$. Acesso em: 8 out. 2012. doi: 10.1001/ jama.2009.1535.

DAY, T.K. Blood gas analysis. Veterinary Clinics of North America: Small Animal Practice, v.32, p.1031-1048, 2002. 
Disponível em: <http://europepmc.org/abstract/MED/12380163>. Acesso em: 8 out. 2012. doi: 10.1016/S0195-5616(02)00035-9.

FERREIRA, M.L.G. et al. Comparação entre as gasometrias venosa e arterial em cães. Revista Brasileira Medicina Veterinária, v.27, p.40-44, 2005.

GOMES, W.J. et al. Artificial respiration with extracorporeal membrane oxygenation in arteriovenous position without pump: experimental study. Arquivos Brasileiros de Cardiologia, v.61, p.93-97, 1993. Disponível em: <http://europepmc.org/abstract/ MED/8297228>. Acesso em:10 out. 2012.

KOPP, R. et al. Role of extracorporeal lung assist in the treatment of acute respiratory failure. Minerva Anestesiologica, v.72, p.587-595, 2006. Disponível em: <http://www minervamedica.it/en/journals/minerva-nestesiologica/article. php?cod=R02Y2006N06A0587>. Acesso em: 8 out. 2012. .

MUELLENBACH, R.M. et al. Arteriovenous extracorporeal lung assist as integral part of a multimodal treatment concept: a retrospectiveanalysis of 22 pacients with ARDS refractory to standard care. European Journal of Anaesthesiology, v.25, p.897-904, 2008. Disponível em: <http://journals.cambridge.org/ action $/$ displayAbstract? fromPage $=$ online $\&$ aid $=2400808>$. Acesso em: 8 out.2012. doi: 10.1017/S0265021508004870.

NEHRA, D. et al. Extracorporeal membrane oxygenation for nonneonatal acute respiratory failure - The Massachusetts General Hospital experience from 1990 to 2008. Archives of Surgery, v.144, p.427-432, 2009. Disponível em: <http://archsurg. jamanetwork.com/article.aspx?articleid $=404945>$. Acesso em: 8 out. 2012. doi:10.1001/archsurg.2009.45.

PERALTA, R. et al. Extracorporeal membrane oxygenation and $\mathrm{CO}_{2}$ removal in an adult after near drowning. Journal of Extra- corporeal Technology, v.37, p.71-74, 2005. Disponível em: $<$ http://www.ncbi.nlm.nih.gov/pubmed/15804162>. Acesso em: 8 out. 2012

PETROU, S. et al. Cost-effectiveness of neonatal extracorporeal membrane oxygenation based on 7-years results from the United Kingdom collaborative ECMO trial. Pediatrics, v.117, p.16401649, 2006. Disponível em: <http://pediatrics.aappublications. org/content/117/5/1640.full.pdf $>$. Acesso em: 8 out. 2012. doi: 10.1542/peds. $2005-1150$

TOTAPALLY, B.R. et al. Cardiovascular stability during arteriovenous extracorporeal therapy: a randomized controlled study in lambs with acute lung injury. Critical Care, v.8, p.r495-r503, 2004. Disponível em: < http://ccforum.com/content/ pdf/cc2983.pdf $>$. Acesso em: 8 out. 2012. doi: 10.1186/cc2983.

ZICK, G. et al. Oxygenation effect of interventional lung assist in a lavage model of acute lung injury: a prospective experimental study. Critical Care, v. 10, p.r56, 2006. Disponível em: <http:// ccforum.com/content/pdf/cc4889.pdf $>$. Acesso em: 20 jun. 2009. doi: $10.1186 / \operatorname{cc} 4889$.

ZIMMERMANN, M. et al. From Baghdad to Germany: use of a new pumpless extracorporeal lung assist system in two severely injured US soldiers. American Society of Artificial Internal Organs Journal, v.53, p.e4-e6, 2007. Disponível em: <http:// journals.lww.com/asaiojournal/pages/articleviewer.aspx?year $=200$ $7 \&$ issue $=05000 \&$ article $=00025 \&$ type $=$ abstract $>$. Acesso em: 27 dez. 2009. doi: 10.1097/MAT.0b013e3180574b37.

ZIMMERMANN, M. et al. Interhospital transportation of patients with severe lung failure on pumpless extracorporeal lung assist. British Journal of Anaesthesia, v.96, p.63-66, 2006. Disponível em: $<$ http://bja.oxfordjournals.org/content/96/1/63.full.pdf + html $>$. Acesso em: 8 out. 2012. doi: 10.1093/bja/aei274. 